\title{
Lateral correlation of multivalent counterions is the universal mechanism of charge inversion
}

\author{
T. T. Nguyen, A. Yu. Grosberg, and B. I. Shklovskii \\ Department of Physics, University of Minnesota, 116 Church St. Southeast, Minneapolis, Minnesota 55455
}

\begin{abstract}
We review works on screening of a macroion, such as colloidal particle or double helix DNA, by multivalent counterions. Multivalent metal ions, charged micelles, short or long polyelectrolytes can play the role of multivalent counterions. Due to the strong Coulomb repulsion such multivalent counterions form a strongly correlated liquid resembling a Wigner crystal at the surface of the macroion. Even if the macroion is neutralized by this liquid, a newly arriving counterion creates in the liquid a correlation hole or image which attracts the ion to the surface. As a result, total charge of adsorbed counterions can substantially exceed the bare macroion charge, so that the macroion net charge inverts its sign. We discuss two previously suggested explanations of charge inversion and show that physics underlying both of them is intrinsically that of correlations, so that correlation is the universal force driving charge inversion.
\end{abstract}

\section{INTRODUCTION}

Charge inversion is a counterintuitive phenomenon in which a strongly charged particle (a macroion) binds so many counterions that its net charge changes sign. As shown below the binding energy of a counterion with large charge $Z$ is larger than $k_{B} T$, so that this net charge is easily observable: it is the net charge that determines a particle drift in a weak field electrophoresis. Charge inversion is possible for a variety of macroions, ranging from the charged surface of mica to charged lipid membranes, colloids, DNA or actin. Multivalent metal ions, small colloidal particles, charged micelles, short or long polyelectrolytes including DNA can play the role of multivalent counterions. Recently charge inversion has attracted significant attention [1] 22].

Theoretically, charge inversion can be also thought of as an over-screening. Indeed, the simplest screening atmosphere, familiar from the linear Debye-Hückel (DH) theory, compensates at any finite distance only a part of the macroion charge. It can be proven that this is true also in the non-linear Poisson-Boltzmann (PB) theory. The statement that the net charge preserves sign of the bare charge agrees with the common sense. One can think that this statement is even more universal than results of $\mathrm{PB}$ equation. However, this presumption of common sense fails for screening by $Z$-valent counterions with large $Z$ ( $Z$-ions). In this case, most of counterions are localized at the very surface of macroion. The energy of their lateral Coulomb interaction may exceed $k_{B} T$ by an order of magnitude or more. As a result [3, 1 , at the macroion surface $Z$-ions form a two-dimensional (2D) strongly correlated liquid (SCL) with properties resembling a Wigner crystal (WC), which is shown in Fig. 1.

The negative chemical potential of this liquid leads to a correlation induced attraction of $Z$-ions to the surface, which brings more of them to the surface than necessary to neutralize it. Role of correlations of $Z$-ions in another physical phenomenon attraction of two likely- charged surfaces was recognized even earlier 23].

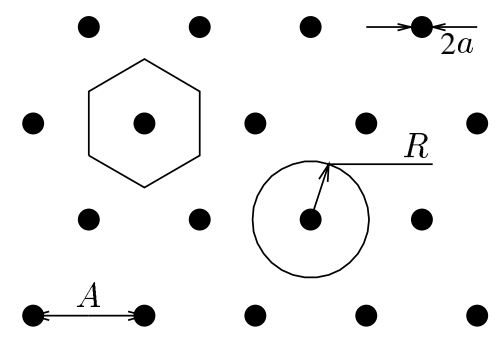

FIG. 1. The Wigner crystal of positive $Z$-ions on the negative uniform background of surface charge. A hexagonal Wigner-Seitz cell and its simplified version as a disk with radius $R$ are shown.

This paper consists of two parts. In the first part, we review recent works on correlation induced charge inversion. Sec. II deals with small $Z$-ions, while in Sec. III the role of $Z$-ions is played by polyelectrolytes. In the second part (Sec. IV), we discuss proposed alternative mechanisms of charge inversion: metallization approach (mental smearing of $Z$-ions on the macroion surface) and counterion release. It is shown there that they are also based on the correlation physics, so that some of the apparent difference is purely semantic.

\section{CORRELATIONS OF SMALL SIZE MULTIVALENT COUNTERIONS}

Let us consider screening a macroion surface with a negative charge density $-\sigma$ by $Z: 1$ salt with positive $Z$-ions in the case of large enough $Z$ and $\sigma$. If such a surface is neutralized by $Z$-ions, the average distance between them in directions parallel to the plane equals $R_{0}=(\pi \sigma / Z e)^{-1 / 2}$. If this distance is much larger than the Gouy-Chapman length $\lambda=D k_{B} T /(2 \pi Z e \sigma)$, which is the typical distance to the surface mean field PB approach obviously fail. The ratio of these two lengths $R_{0} / \lambda=2 \Gamma$, where $\Gamma=Z^{2} e^{2} /\left(R_{0} D k_{B} T\right)$ is the dimen- 
sionless inverse temperature measured in units of the typical interaction energy and $D$ is the dielectric constant of water. Here we are concerned with large enough $Z$ and $\sigma$ at which parameter $\Gamma \gg 1$. For example, at $Z=3$ and $\sigma=1.0 \mathrm{e} / \mathrm{nm}^{2}$ we get $\Gamma=6.4, \lambda \simeq 0.08 \mathrm{~nm}$ and $R_{0} \simeq 1.0$ $\mathrm{nm}$. In such conditions Gouy-Chapman solution of $\mathrm{PB}$ equation can not be valid any more. Indeed, if distance of a $Z$-ion to the surface $x$ is in the interval $\lambda \ll x \ll R_{0}$, it does not feel other $Z$-ions but interacts only with the macroion surface. This interaction leads to an exponential decay of concentration of $Z$-ions as function of $x$ in the range $x \ll R_{0}$ instead of $1 / x^{2}$ Gouy-Chapman law. A new theory based on inequality $\Gamma \gg 1$ was suggested in Ref. [3]4]. The main idea of this theory is that at $\Gamma \gg 1$ the screening atmosphere can be divided in two distinct phases: 2D SCL, which should be treated exactly, and the gas phase which at large distances $x$ can be desribed by the PB equation and is in equilibrium with 2D SCL.

Thermodynamic properties of 2D liquid of classical charged particles on the neutralizing background, so called one-component plasma (OCP) are well known (see bibliography in Ref. [3:4). The chemical potential of OCP can be written as $\mu=\mu_{i d}+\mu_{c}$, where $\mu_{i d}$ and $\mu_{c}$ are its ideal and correlation parts. At $T=0$ OCP forms WC. To calculate $\mu_{c}$, we start from the energy of WC per $Z$-ion,

$$
\varepsilon(n) \simeq-1.11 Z^{2} e^{2} / R D=-1.96 n^{1 / 2} Z^{2} e^{2} / D,
$$

where $R=(\pi n)^{-1 / 2}$ is the radius of its Wigner-Seitz (WS) cell (See Fig. 1) and $n$ is $2 \mathrm{D}$ concentration of $Z$ ions. This gives $\mu_{c}=\mu_{W C}$, where

$$
\mu_{W C}=\frac{\partial[n \varepsilon(n)]}{\partial n}=-1.65 \Gamma k_{B} T=-1.65 Z^{2} e^{2} / D R .
$$

In the range of temperatures where $1 \ll \Gamma \ll 130 \mathrm{WC}$ is melted and for SCL $\mu_{c}=\mu_{W C}+\delta \mu$, where $\delta \mu$ is a small positive correction [3, 1 .

Physics of a large and negative $\mu_{W C}$ can be understood as follows. Let us imagine for a moment that an insulating macroion is replaced by a neutral metallic particle. In this case, each $Z$-ion creates an image charge of opposite sign inside the metal. Energy of attraction to the image is $U(x)=-(Z e)^{2} / 4 D x$, where $x$ is the distance from $Z$ ion to the surface. At the metal surface energy of the interaction with the image equals $U(a)=-(Z e)^{2} / 4 D a$, where $a$ is radius of the counterion. The chemical potential $\mu_{W C}$ for a charged surface of an insulating macroion can be interpreted in a similar language of images.

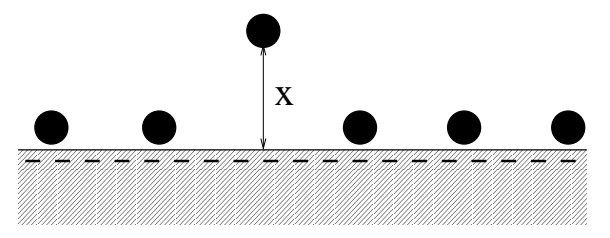

FIG. 2. The origin of attraction of a new positive $Z$-ion to the already neutralized surface. $Z$-ions are shown by solid circles. The new $Z$-ion creates its negative correlation hole.

Consider bringing a new $Z$-ion to the macroion surface already covered by an adsorbed layer of $Z$-ions (See Fig. $2)$. This layer plays the role of a metal surface. Indeed, the new $Z$-ion repels nearest adsorbed ones, creating a correlation hole for himself. In other words, it creates a negative image. Calculation of the energy of attraction to the image in this case is, however, less trivial than in the case of a metal. The problem is that minimal size of the image in the adsorbed layer is equal to the WS cell radius $R$. (The adsorbed layer is a good metal only at larger scales.) Thus, for WC we arrive at $\mu_{W C} \sim-(Z e)^{2} / D R$. Eq. (2) provides the numerical coefficient in this expression. It is clear now that contrary to the case of a metallic surface, charge inversion for insulating macroion requires a finite density of an adsorbed layer, and, correspondingly, a finite bare charge density $\sigma$.

Correlation induced attraction of $Z$-ion to a neutral SCL has two interesting analogies in the solid state and atomic physics. The energy $\left|\mu_{W C}\right|$ plays the same role for $Z$-ions as the work function plays for electrons of a metal or the ionization energy plays in a many-electron atom. It is known that in the Thomas-Fermi approximation which neglects exchange and correlation holes, both the work function of a metal 24 and the ionization energy of a many-electron atom 25] vanish. For screening by $Z$-ions the PB approximation is an analog of the Thomas-Fermi approximation and it results in $\mu_{c}=0$. Only correlations lead to a finite work function or ionization energy and to a finite $\mu_{c} \simeq \mu_{W C}$.

Let us now discuss behavior of the concentration of $Z$-ions, $N(x)$ near the surface. For this purpose let us extract a $Z$-ion from SCL and move it along the $x$ axis. As it is mentioned above, this $Z$-ion leaves behind its correlation hole. In the range of distances $x \ll R$, the correlation hole is a disc of the surface charge with radius $R$ (WS cell) and the $Z$-ion is attracted to the surface by its uniform electric field $E=2 \pi \sigma / D$. We can say that our $Z$-ion does not see other $Z$-ions until $x \ll R$. Therefore, $N(x)=N_{s} \exp (-x / \lambda)$ at $x \ll R$. Here $N_{s}=n / \lambda$ is the three-dimensional concentration of $Z$-ions at the plane. At large enough $x$ the concentration $N(x)$ should saturate at the level of

$$
N_{0}=N_{s} \exp \left(-\left|\mu_{W C}\right| / k_{B} T\right)=N_{s} \exp (-1.65 \Gamma) .
$$

Let us find out how and where this saturation happens. At $x \sim R=2 \Gamma \lambda$ the ratio $x / \lambda$ reaches roughly speaking a half of $\left|\mu_{W C}\right| / k_{B} T$. At $x \gg R$ the $Z$-ion creates the image with relatively small radius $R$ which attracts $Z$-ion back and provides the decaying Coulomb correction to the activation energy of $N(x): N(x)=$ 
$N_{s} \exp \left(-\left[\left|\mu_{W C}\right|-Z^{2} e^{2} / 4 D x\right] / k_{B} T\right)$. This correction is similar to the well known "image" correction to the work function of a metal [24]. At $x=Z^{2} e^{2} / 4 D k_{B} T=\lambda \Gamma^{2} / 2$ this correction reaches $k_{B} T$, so that $N(x)$ saturates at the value $N_{0}$.

The dramatic difference between the described above exponential decay of $N(x)$ and the Gouy-Chapman $1 /(\lambda+x)^{2}$-law is obviously related to the correlation effects. Recently such an exponential decay of $N(x)$ with the following tendency to saturation was rederived in more formal way and confirmed by Monte-Carlo simulations [26]. At distances $x \gg \lambda \Gamma^{2} / 2$, interaction of the removed ion with its correlation hole in SCL is not important and the correlation between ions of the gas phase are even weaker because $N(x)$ is exponentially small. Therefore, at larger distances one can describe $N(x)$ by $\mathrm{PB}$ equation [3. [4].

Studying charge inversion, we want to calculate the net charge density of the macroion surface $\sigma^{*}=-\sigma+Z e n>$ 0 . Even though the system is not neutral, we can still use the chemical potential of a neutral OCP given by Eq. (2) after the following exact transformation. Indeed, let us add uniform charge densities $-\sigma^{*}$ and $\sigma^{*}$ to the macroion surface. The first addition results in a neutral OCP. The second addition creates only uniform potential $e \psi(0)$ on the macroion surface. For example, if the macroion is a sphere with radius $r$ and screening radius of solution is larger than $r$ we get $\psi(0)=Q^{*} / D r$, where $Q^{*}=4 \pi r^{2} \sigma^{*}=-Q+4 \pi r^{2} n e Z$ is the net charge of the sphere and $-Q=4 \pi r^{2} \sigma$ is its bare charge. It is important to emphasize that macroscopic net charge $Q^{*}$ does not interact with OCP, because potential of the uniformly charged sphere at the neutral OCP is constant.

The condition of balance of the electrochemical potential at the surface of macroion, $\mu$, and in the bulk of solution, $\mu_{b}$ now can be written as $Z e \psi(0)=-\mu_{W C}+$ $\left(\mu_{b}-\mu_{i d}\right)=-\mu_{W C}+k_{B} T \ln \left(N_{s} / N\right)=k_{B} T \ln \left(N / N_{0}\right)$, where $N$ is the concentration of $Z$-ions in the bulk of solution. It is clear that when $N>N_{0}$, the net charge density $\sigma^{*}$ is indeed positive, i.e. has the sign opposite to the bare charge density $-\sigma$. The concentration $N_{0}$ is very small because $\left|\mu_{W C}\right| / k_{B} T=1.65 \Gamma \gg 1$. Therefore, it is easy to achieve charge inversion increasing $N$. At large enough $N$ we have $\left|\mu_{b}-\mu_{i d}\right| \ll\left|\mu_{W C}\right|$. This gives a simple equation for calculation of the maximal inverted charge density

$$
\psi(0)=\left|\mu_{W C}\right| / Z e .
$$

Let us consider a sphere with charge $-Q$ screened by $Z: 1$ salt with a large concentration, $N$. In this case $\psi(0)=Q^{*} / D r$ and Eq. (4) has a simple meaning: $\left|\mu_{W C}\right| / Z e$ is the "correlation" voltage which charges a spherical capacitor. Expressing $R$ and $\left|\mu_{W C}\right|$ through $Q$ and $Z$ we arrive at the simple prediction [4 for the maximum possible inverted charge:

$$
Q^{*}=0.83 \sqrt{Q Z e}
$$

This charge is much larger than $Z e$, but still is smaller than $Q$ because of limitations imposed by the large charging energy of the macroscopic net charge. For example, for $Q=100 e, Z=4$, we get $Q^{*}=17 e$. Eq. (5) was recently confirmed by numerical simulations [15,22].

It was also shown in Ref. [3: th, that in the case of a cylinder, the conventional picture of nonlinear screening known as the Onsager-Manning (OM) condensation should be strongly modified when dealing with multivalent ions. Consider a cylinder with a negative linear charge density $-\eta$ and assume that $\eta>\eta_{z}$, where $\eta_{z}=k_{B} T D / Z e$. OM theory [27], shows that such a strongly charged cylinder is partially screened by counterions residing at its surface, so that net linear charge density of the cylinder, $\eta^{*}=-\eta_{z}$. The rest of the charge is screened at much larger distances according to the linear DH theory. The OM theory uses PB mean field approach and, therefore, does not take into account lateral correlations of counterions. It is shown in Ref. [3, th that the correlation induced negative chemical potential $\mu_{W C}$ leads to inversion of the sign of $\eta^{*}$ at $N>N_{0}$ in the case of cylinder, too. At large enough $N$ inverted charge density $\eta^{*}$, can reach $k_{B} T D / e$.

Even stronger charge inversion of a spherical or cylindrical macroion can be obtained in the presence of substantial concentration of monovalent salt, such as $\mathrm{NaCl}$, in solution. Monovalent salt screens long range Coulomb interactions stronger than short range lateral correlations between adsorbed $Z$-ions. Therefore, screening by monovalent salt diminishes the charging energy of the macroion much stronger than the correlation energy of $Z$-ions. As a results, the inverted charge $Q^{*}$ becomes larger than that predicted by Eq. (5) and scales linearly with $Q$. Since, in the presence of a sufficient concentration of salt, the macroion is screened at the distance smaller than its size, the macroion can be thought of as an over-screened surface, with inverted charge $Q^{*}$ proportional to the surface area. In this sense, overall shape of the macroion and its surface is irrelevant, at least to a first approximation. Therefore, we consider here a simple case: screening of a planar macroion surface with a negative surface charge density $-\sigma$ by solution with concentration $N$ of $Z: 1$ salt and a large concentration $N_{1}$ of a monovalent salt. Correspondingly, we assume that all weak interactions are screened with DH screening length $r_{s}=\left(8 \pi l_{B} N_{1}\right)^{-1 / 2}$. For simplicity, we discuss here only a charged surface of insulating macroion with the same dielectric constant as water. Complications related to the difference between dielectric constants are discussed in Ref. [13].

The dependence of the charge inversion ratio, $\sigma^{*} / \sigma$, on $r_{s}$ was calculated analytically [13] in two limiting cases $r_{s} \gg R_{0}$ and $r_{s} \ll R_{0}$, where $R_{0}=(\pi \sigma / Z e)^{-1 / 2}$ is the radius a WS cell at the neutral point $n=\sigma / Z e$. At 
$r_{s} \gg R_{0}$ calculation starts from Eq. (44). Electrostatic potential can be calculated as potential of the plane with the charge density $\sigma^{*}$ screened at the distance $r_{s}$. This gives $\psi(0)=4 \pi \sigma^{*} r_{s}$. At $r_{s} \gg R_{0}$ screening by monovalent ions does not change Eq. (2) substantially so that we still can use it in Eq. (四) which now describes charging of a plane capacitor by voltage $\left|\mu_{W C}\right| / Z e$. This gives [13]

$$
\sigma^{*} / \sigma=0.41\left(R_{0} / r_{s}\right) \ll 1 \quad\left(r_{s} \gg R_{0}\right) .
$$

Thus, at $r_{s} \gg R_{0}$ inverted charge density grows with decreasing $r_{s}$. Now we switch to the case of strong screening, $r_{s} \ll R_{0}$. It seems that in this case $\sigma^{*}$ should decrease with decreasing $r_{s}$, because screening reduces the energy of SCL and leads to its evaporation. In fact, this is what eventually happens. However, there is a range where $r_{s} \ll R_{0}$, but the energy of SCL is still large. In this range, as $r_{s}$ decreases, the repulsion between $Z$-ions becomes weaker and makes it easier to pack more $Z$-ions on the plane. Therefore, $\sigma^{*}$ continues to grow with decreasing $r_{s}$. At $r_{s} \ll R_{0}$ it is convenient to minimize directly the free energy of $\mathrm{WC}$ of $Z$-ions at the macroin surface with respect of $n$. This free energy consists of nearest neighbor repulsion energies of $Z$-ions and the attraction energy of $Z$-ions to the charge surface. All interactions are screened according to DH theory, so that interaction of non-nearest neighbors can be neglected. This gives 13.

$$
F=-4 \pi \sigma r_{s} Z e n / D+\left(3 n Z^{2} e^{2} / D A\right) \exp \left(-A / r_{s}\right),
$$

where $A=(2 / \sqrt{3})^{1 / 2} n^{-1 / 2}$ is the lattice constant of the hexagonal WC. Minimizing this free energy with respect to $n$ one arrives at

$$
\sigma^{*} / \sigma=(\pi / 2 \sqrt{3})\left[R_{0} / r_{s} \ln \left(R_{0} / r_{s}\right)\right]^{2} \quad\left(r_{s} \ll R_{0}\right) .
$$

Thus $\sigma^{*} / \sigma$ grows with decreasing $r_{s}$ and can become larger than $100 \%$. At $r_{s} \sim R_{0}$ Eq. (8) and Eq. (6) match each other.

\section{CORRELATIONS OF ADSORBED POLYELECTROLYTE MOLECULES}

\section{A. Adsorption of rod-like polyelectrolytes on the charged plane}

A practically important class of multivalent ions are polyelectrolyte (PE) molecules. In this section we discuss charge inversion caused by adsorption of long rod-like $Z$ ions. To make signs consistent with the case of DNA, we assume that PE charge is negative, $-\eta_{0}$ per unit length, while the macroion surface is a plane with positive charge density $\sigma$. The basic idea is once again the strong lateral correlations: due to the strong lateral repulsion, charged rods adsorbed at the surface tend to be parallel to each other and have a short range order of an one-dimensional $\mathrm{WC}$ in the perpendicular to rods direction (Fig. 3).

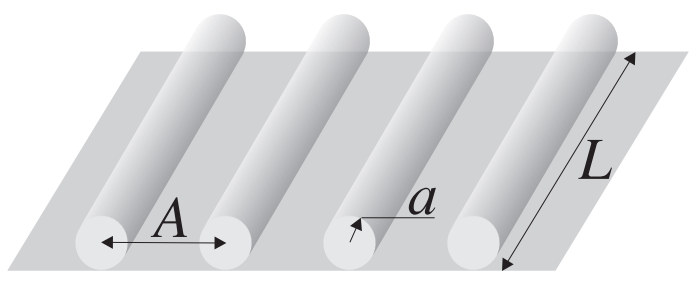

FIG. 3. Rod-like negative $Z$-ions such as double helix DNA are adsorbed on a positive uniformly charged plane. Strong Coulomb repulsion of rods leads to one-dimensional crystallization with lattice constant $A$.

It is well known that the bare charge density of DNA, $-\eta_{0}$, is about four times the critical density $-\eta_{c}=k_{B} T / e$ of OM condensation. Therefore, about three quarters of the bare charge of an isolated DNA is compensated by positive monovalent ions residing at its surface so that the net charge of DNA in the bulk solution is $\eta^{*}=-\eta_{c}$. This is generally not true for adsorbed DNA. Indeed, some OM-condensed ions, as they are repelled from the charged surface, can be pushed away from DNA and released into the solution when DNA is adsorbed [6]. This effect is particularly important when monovalent salt concentration is low, otherwise counterions do not gain enough entropy to justify the release.

It was shown 13 that the criterion of counterion release is governed by the comparison of screening length, $r_{s}$, of monovalent salt and the spacing between critically charged rods, $A_{0}=\eta_{c} / \sigma$, when they neutralize the plane. If screening is strong and $r_{s} \ll A_{0}$, the potential of the surface is so weak that counterions are not released. In other words, charge density for each adsorbed DNA helix remains $\eta^{*}=-\eta_{c}$. Simultaneously, at $r_{s} \ll A_{0}$ the DH approximation can be used to describe screening of the charged surface by monovalent salt. Using these simplifications one can directly minimize the free energy of one-dimensional crystal of DNA rods on the positive surface written similarly to Eq. (7). Then the competition between attraction of DNA helices to the surface and the repulsion of the neighboring helices results in the negative net surface charge density $-\sigma^{*}$. The charge inversion ratio reads [9, 13] :

$$
\sigma^{*} / \sigma=\left(\eta_{c} / \sigma r_{s}\right) / \ln \left(\eta_{c} / \sigma r_{s}\right) \quad\left(\eta_{c} / \sigma r_{s} \gg 1, r_{s} \ll A_{0}\right) .
$$

Thus the inversion ratio grows with decreasing $r_{s}$ as in the case of spherical $Z$-ions. At small enough $r_{s}$ and $\sigma$, the inversion ratio can reach $200 \%$ before DNA molecules are released from the surface. It is larger than for spherical ions, because in this case, due to the large length of DNA helix, the correlation energy remains large and 
WC-like short range order is preserved at smaller values of $\sigma$ and/or $r_{s}$. In the works [13,14], we called this phenomenon "giant charge inversion."

Let us switch now to the opposite extreme of weak screening by a monovalent salt, $r_{s} \gg A_{0}$. In this case, screening of the overcharged plane by monovalent salt becomes strongly nonlinear, with the Gouy-Chapman screening length $\lambda^{*}=D k_{B} T /\left(2 \pi e \sigma^{*}\right)$ much smaller than $r_{s}$. Furthermore, some counterions are released from DNA upon adsorbtion. As a result the absolute value of the net linear charge density of each adsorbed DNA, $\eta^{*}$, becomes larger than $\eta_{c}$. Two nonlinear equations for $\sigma^{*}$ and $\eta^{*}$ are derived in Ref. 13 and are discussed below in Sec. IV. Their solution at $r_{s} \gg A_{0}$ reads:

$$
\begin{gathered}
\sigma^{*} / \sigma=\left(\eta_{c} / 2 \pi a \sigma\right) \exp \left(-\sqrt{\ln \left(r_{s} / a\right) \ln \left(A_{0} / 2 \pi a\right)}\right), \\
\eta^{*}=\eta_{c} \sqrt{\ln \left(r_{s} / a\right) / \ln \left(A_{0} / 2 \pi a\right)} .
\end{gathered}
$$

At $r_{s} \simeq A_{0}$ we get $\eta^{*} \simeq \eta_{c}, \lambda^{*} \simeq r_{s}$ and $\sigma^{*} / \sigma \simeq$ $\eta_{c} /\left(2 \pi r_{s} \sigma\right)$ so that Eq. (10) crosses over smoothly to the strong screening result of Eq. (9). Since $\eta^{*}$ can not be smaller than $\eta_{c}$, the fact that $\eta^{*} \simeq \eta_{c}$ already at $r_{s} \simeq A_{0}$ proves that at $r_{s} \ll A_{0}$, indeed, $\eta^{*} \simeq \eta_{c}$. Simultaneously, the fact that at this point $\lambda^{*} \simeq r_{s}$ means that $r_{s} \ll \lambda^{*}$ at $r_{s} \ll A_{0}$, so that screening in this regime becomes linear and is described by DH theory.

\section{B. Flexible polyelectrolytes wrapping around the charged spheres}

Until now we talked only about adsorption of a rodlike $\mathrm{PE}$. If the persistence length $L_{p}$ is finite, $\mathrm{PE}$ is released from the charged surface when $r_{s}$ is very small. This happens when interaction of a PE segment of the length $L_{p}$ with the surface becomes smaller than $k_{B} T$. On the other hand, at larger $r_{s}$ a flexible PE lays flat at the surface. Due to its electrostatic rigidity neighboring molecules form WC-like SCL and behave similarly to that of a rod-like PE. The correlation induced adsorption of a flexible weakly charged PE on an oppositely charged surface was comprehensively studied in Ref. [19]. When surface is covered by less than one complete layer of $\mathrm{PE}$, results are indeed close to presented above.

Let us also mention the charge inversion in the problem of complexation of a positive sphere and a flexible negative PE (Fig. 4). Refs. [5, 6, 8, 10] predicted substantial charge inversion in this case: more PE is wound around a sphere than necessary to neutralize it. Role of correlations in such charge inversion was recently emphasized in Ref. [16]. It was shown that neighbouring turns repel each other and form almost equidistant solenoid, which locally resembles WC. The tail of PE repels PE adsorbed at the surface and creates a correlation hole, which attracts the tail back to the surface.

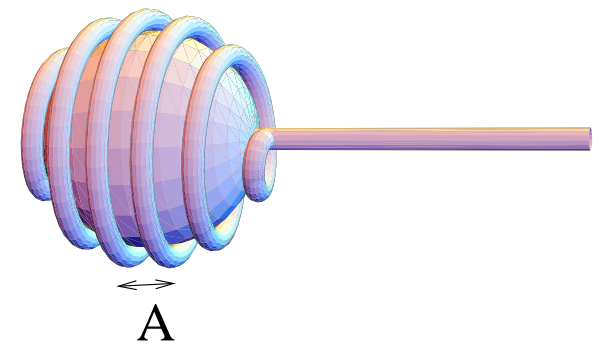

FIG. 4. A PE molecule winding around a spherical macroion. Due to Coulomb repulsion, neighboring turns lie parallel to each other. Locally, they resemble a one-dimensional Wigner crystal with the lattice constant $A$.

The last example of the correlation driven charge inversion we want to mention here is the complexation in a solution with given concentrations of a long flexible negative $\mathrm{PE}$ and positive spherical particles such as colloids, micelles, globular proteins or dendromers 18]. PE binds spheres winding around them. If the total charge of $\mathrm{PE}$ in the solution is larger than the total charge of spheres, repulsive correlation of $\mathrm{PE}$ turns on a sphere surface lead to inversion of the net charge of each sphere. Negative spheres repel each other and form on the PE molecule periodic beads-on-a-string structure, which resembles 10 nm fiber of chromatin (Fig. 5).

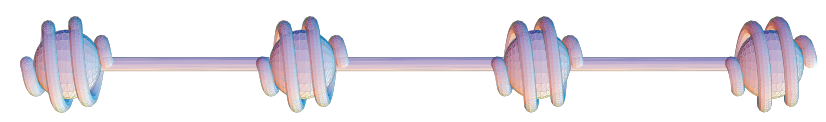

FIG. 5. Complexation of a negative PE molecule and many positive spheres in a necklace-like structure. On the surface of a sphere neighboring PE turns form WC similar to Fig. 4. At larger scale, charged spheres repel each other and form one-dimensional Wigner crystal along the PE molecule.

If the total charge of $\mathrm{PE}$ is smaller than the total charge of spheres, the latter are under-screened by PE and their net charges are positive. Bound spheres once more repel each other and form a periodic necklace. Because a segment of PE wound around a sphere interacts almost exclusively with this sphere, it plays the role of WS cell. These WC-like correlations lead to inversion of charge of a PE molecule: spheres bind to PE in such a great number that the net charge of the PE molecule becomes positive. It is shown in Ref. 18 that inverted charge of PE by the absolute value can be larger than the bare charge of PE even in the limit of very weak screening by monovalent salt. When a large concentration of a monovalent salt is added charge inversion can reach giant proportions. This theory is in a qualitative agreement with recent experiments on micelles-PE systems [11]. 


\section{ARE THERE OTHER MECHANISMS OF CHARGE INVERSION?}

However briefly, we completed the review of works on correlation induced charge inversion. It is now time to ask: Are there alternative, correlation-independent, electrostatic mechanisms leading to this phenomenon? Our answer is no, and we argue that correlations-based mechanism is the universal one. Specifically, we consider below two theories suggested in literature. One, pioneered by [5.7], considers adsorbed $Z$-ions as a smeared continuous medium similar to a metal. We argue that this metallization approach is an approximation for correlation theory; we discuss when this anzatz is accurate, and when it fails. The other theory, put forward by [6, views counterion release as the driving force behind charge inversion. To this end, we show that while counterion release is obviously favorable for charge inversion, it is itself driven by correlations.

\section{A. Metallization approach}

This approach was clearly formulated for the cases of complexation of a PE with a sphere in $[5]$ and for adsorption of flexible polymers on a charged plane in [7]. The physical basis of this theory is a single, seemingly benign approximation. Namely, it considers the adsorbed $Z$-ions, at the macroion surface, as a smeared continuum, while still treating bulk solution as consisting of discrete charges. Why is smearing so much favorable as to cause adsorbtion of $Z$-ions to the macroion which is already neutralized or even overcharged?

The answer is that smearing amounts to complete neglect of self-energy $\varepsilon_{\text {self }}=Z^{2} e^{2} / 2 D a$ of adsorbed $Z$-ions; better to say, it neglects the energy of each $Z$-ion's electric field in the range of distances between the ion radius $a$ and macroion size $r$. On the other hand, correlations can be viewed as physical mechanism eliminating a part of self-energy of each $Z$-ion corresponding to the field in the range of distances between correlation hole size $R$ (which plays the role of screening radius of SCL) and macroion size $r$. In this sense, metallization overestimates the role of correlations and the charge inversion ratio, particularly when $R \gg a$.

To appreciate the difference between correlated and smeared set of $Z$-ions, it is worth comparing both to the random distribution of the same ions. Correlated configuration is more favorable than random: correlations happen because ions reconfigure themselves non-randomly to gain some free energy. Smeared continuum is also more favorable than random configuration of discrete ions, the difference being precisely the self-energy of ions.

Let us illustrate these ideas in the simplest geometry - a sphere with charge $-Q$ and radius $r$ screened by spherical $Z$-ions of radius $a \ll r$ in the absence of monovalent salt.
Correlation theory, as we have seen, results in Eq. (5) for this problem. What does metallization approach predicts for the same problem? Assuming $a \ll r$, it is easy to write down all relevant energies: $E_{m e t}=Q^{* 2} / 2 D r$, which corresponds to the uniformly smeared net charge $Q^{*} ; E_{\text {rand }}=E_{\text {met }}+M \varepsilon_{\text {self }}$, which is the energy of the sphere with $M=4 \pi r^{2} n$ randomly distributed $Z$-ions on its surface; and $E_{W C}=E_{\text {rand }}+M \varepsilon(n)$, which is the energy of SCL of $Z$-ions. Here $\varepsilon(n)$ is the energy of WC per $Z$-ion given by Eq. (11). The last term of $E_{W C}$ is the correlation energy [28], it is the negative (favorable) contribution due to the difference between correlated and random configurations of $Z$-ions. As expected, $E_{\text {met }}<E_{W C}<E_{\text {rand }}$.

To address equilibrium charge inversion, let us now balance the chemical potential of $Z$-ions at the surface and in the bulk. Apart from translational entropy contribution negligible at high enough concentrations of $Z$ ions, we have: for the bulk part chemical potential is equal to the self-energy $\varepsilon_{\text {self }}$; and for the surface part $\mu=\partial E / \partial M$, where $E$ is either $E_{m e t}$, or $E_{\text {rand }}$, or $E_{W C}$. Therefore, depending on the approximation we want to use, the equilibrium condition reads

$$
\begin{aligned}
\text { metal : } & \varepsilon_{\text {self }}=Q_{\text {met }}^{*} Z e / D r \\
\text { random }: & \varepsilon_{\text {self }}=Q_{\text {rand }}^{*} Z e / D r+\varepsilon_{\text {self }} \\
\text { SCL }: & \varepsilon_{\text {self }}=Q_{S C L}^{*} Z e / D r+\varepsilon_{\text {self }}-1.65 Z^{2} e^{2} / R D .
\end{aligned}
$$

Looking at the result starting from the correlation theory, we see that self-energies cancel and $Q_{S C L}^{*}=1.65 \mathrm{Zer} / R$, producing Eq. (5). By contrast, random distribution leads to $Q_{\text {rand }}^{*}=0$, i.e., charge inversion is impossible without correlations. Finally, metallization approach yields $Q_{m e t}^{*}=Z e r / 2 a$. Comparing with result for SCL, metallization approach is off by a numerical factor only when $R \sim 2 a$, while it substantially overestimates charge inversion at $R \gg a$.

We can repeat all the above arguments for adsorption of PE of length $L<r$, radius $a$, and charge density below OM threshold, $\eta<\eta_{c}$, on a sphere with radius $r$ and charge $-Q$. At the surface of sphere PE molecules form one of several possible anisotropic WC-like phases. For example, it can be nematic SCL with distance $A$ between almost parallel neighboring PE molecules or a solenoid with distance $A$ between turns, where PE molecules continue each other simulating a long $\mathrm{PE}$ which winds around the sphere. In any case, balancing chemical potentials leads to the equations similar to Eqs. (12):

$$
\begin{aligned}
\text { metal : } & \varepsilon_{\text {self }}=Q_{\text {met }}^{*} \eta L / D r \\
\text { random : } & \varepsilon_{\text {self }}=Q_{\text {rand }}^{*} \eta L / D r+\varepsilon_{\text {self }} \\
\text { SCL : } & \varepsilon_{\text {self }}=Q_{S C L}^{*} \eta L / D r+\varepsilon_{\text {self }}-\left(\eta^{2} / D L\right) \ln (L / A),
\end{aligned}
$$

where $\varepsilon_{\text {self }}=\eta^{2} L D^{-1} \ln (L / a)$. Thus, in the correlation theory, we get $Q_{S C L}^{*}=\left(r \eta^{2} L / Z e^{2}\right) \ln (L / A)$ because of cancellation of self-energies. Random distribution of positions and orientations of PE molecules does 
not lead to charge inversion. For metallized state, we have self-energy only in the bulk, which gives $Q_{m e t}^{*}=$ $\left(r \eta^{2} L / Z e^{2}\right) \ln (L / a)$. In complete analogy with the previous case, we see that metallization approach generally overestimates charge inversion, particularly at $A \gg a$, i.e., when few PE molecules are adsorbed. With growing coverage the metallization approximation becomes increasingly accurate.

Qualitatively new effect, however, becomes important when macroion is so strongly charged that its neutralization requires almost full layer of $Z$-ions. Excluded volume effect of hard cores of $Z$-ions adds a positive term to the chemical potential of SCL, which is proportional to $k_{B} T$ and diverges at the full coverage simultaneously with pressure. Close to the full layer this term compensates and then over-compensates the negative Coulomb term $\mu_{W C}$, so that charge inversion disappears. In the language of images, this happens because a full layer is incompressible and does not allow for correlation hole and image. For even larger macroion charge, the second layer starts to form, launching a new wave of charge inversion. In the beginning, charge inversion is small because all the attraction of a new $Z$-ion approaching the surface is provided by a weak interaction with an inflated image in the emerging second layer, where once again $A \gg a$. Continuing, we arrive at the prediction of oscillating inverted charge $Q^{*}$ as a function of $Q$ (see Fig. 6), where charge inversion vanishes every time when the top layer of $Z$-ions is full 29]. Metallization approximation fails to capture the physics of these oscillations.

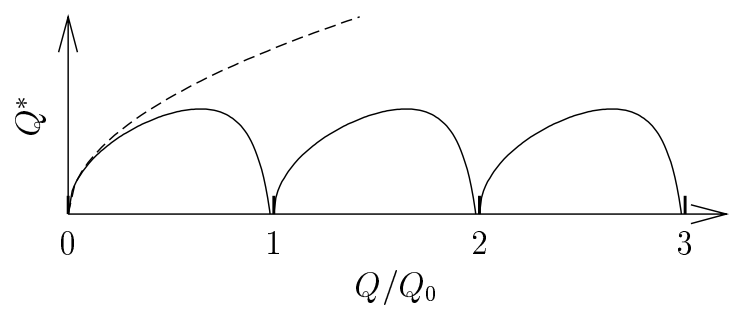

FIG. 6. Schematic plot of the oscillating inverted charge of the sphere $Q^{*}$ as a function of the absolute value $Q$ of its bare charge. Here $Q_{0}$ is the charge of the full layer of $Z$-ions. Dashed line obeys Eq. (3), which is derived for $Z$-ions of vanishing radius $a$.

The oscillations of charge inversion we arrived at are similar to the oscillations of the compressibility and magneto-capacitance in the quantum Hall effect, which are related to consecutive filling of Landau levels [30]. In this sense, we deal with a classical analog of the quantum Hall effect.

We would like to conclude this subsection emphasizing the difference between metallization approach and the PB one. PB approximation effectively smears $Z$-ions everywhere, both at the macroion surface and in the bulk of solution, while the metallization approach keeps $Z$ - ions of the bulk discrete. Therefore, it is not surprising that the metallization approach somewhat overestimates charge inversion, while there is no charge inversion in $\mathrm{PB}$ approximation.

\section{B. Counterion release}

Consider now adsorbtion of DNA, or other polymer charged above the OM threshold $\eta_{c}$. The result of correlation theory for this case is presented in Sec. III. Another, seemingly independent reason for charge inversion, was suggested in [6], arguing that the entropy gain of counterions released by DNA drives charge inversion. Of course, this theory does not pretend for universality, as it applies to highly charged polymers only. Nevertheless, we want to understand the relations of counterion release and correlations theories.

Let us start with a simple qualitative argument. Imagine that neutralizing DNA molecules, along with their OM-condensed small ions, are adsorbing on the macroion (possibly releasing some of the ions), and currently the neutralization condition is achieved. Assume further that DNA rods are distributed randomly, uncorrelated in both positions and orientations. In this case, next arriving DNA molecule feels no average field, so that it has no reason to release its counterions. The situation is completely different if DNA molecules are correlated on the surface, where locally each molecule is surrounded by a correlation hole - positive stripe of the background charge (WS cell). The corresponding field, or positive potential of the WS cell, may cause release of counterions not only at the neutrality point, but even if the surface overall is overcharged $(\psi(0)<0)$. In other words, correlation hole, or adjustment of DNA molecules to each other, or image charge, or correlations (all synonyms!) is a necessary condition for both counterion release and charge inversion.

To understand this better, let us re-examine the fundamental physical conditions governing the adsorbtion equilibrium, namely that chemical potentials of both DNA and small ions should be the same at the surface of macroion and in the bulk of the solution. The equations expressing these conditions were derived in [14 (Eqs. (57) and (60) of that work). We re-derive them below, as Eq. (15) and Eq. (16). We deal below only with the case of weak screening, when $r_{s} \gg A$.

Consider first chemical potential of small ions. It must be the same for ions in the bulk solution, ions condensed on the surface of dissolved DNA rods, and ions on those DNA rods which, in turn, are adsorbed on the macroion. The two corresponding equations read:

$$
\begin{aligned}
& k_{B} T \ln \left(N_{1, s b} / N_{1}\right)=\left(2 e \eta_{c} / D\right) \ln \left(r_{s} / a\right) \\
& k_{B} T \ln \left(N_{1, s s} / N_{1}\right)=-e \psi(0)+e\left(2 e \eta^{*} / D\right) \ln (A / 2 \pi a) .
\end{aligned}
$$


The left-hand sides of Eqs. (14) is obviously the entropy loss of monovalent ions when they move from the solution to the surface of DNA and the right-hand side is the potential energy gain per ion. In the entropic part, $N_{1}$, $N_{1, s b}$ and $N_{1, s s}$ are the concentrations of counterions in the bulk and at the surface of the dissolved and adsorbed DNA, respectively. In the energetic part, $\psi(0)$ is the average potential of the uniformly over-charged plane with charge density $\sigma^{*}$, while logarithmic terms correspond to the potential of DNA having charge density $-\eta_{c}$ in the bulk, where it is screened by monovalent ions at the distance $r_{s}$, and DNA with charge density $-\eta^{*}$ at the surface, where it is screened by other DNA molecules at the distance $A / 2 \pi$. As regards $\psi(0)$, it is given by the Gouy-Chapman formula $\psi(0) \simeq-\left(2 k_{B} T / e\right) \ln \left(\left(r_{s}+\right.\right.$ $\left.\left.\lambda^{*}\right) / \lambda^{*}\right)$, where $\lambda^{*}=D k_{B} T /\left(2 \pi e \sigma^{*}\right)$, because plane as a whole is non-linearly screened. Concentrations $N_{1, s s}$ and $N_{1, s b}$ are related to the corresponding net charges: $N_{1, s s} / N_{1, s b}=\left(\eta_{0}-\eta^{*}\right) /\left(\eta_{0}-\eta_{c}\right)$. According to Eq. (11) $\eta^{*}$ differs only logarithmically from $\eta_{c}$. Therefore, we can neglect $\ln \left(N_{1, s s} / N_{1, s b}\right)$ and use the same notation $N_{1, s}$ for both concentrations. Taking into acount that $\eta_{c}=k_{B} T D / e$, we get

$$
\eta_{c} \ln \left(\lambda^{*} / a\right)=\eta^{*} \ln (A / 2 \pi a) .
$$

Already at this stage we can look at what happens if we neglect correlations between adsorbed DNA rods. In this case, they are screened by small ions only, and the corresponding screening length is $\lambda^{*}$ instead of $A / 2 \pi$. Eq. (15) then gives $\eta^{*}=\eta_{c}$. Thus, if adsorbed DNA molecules were uncorrelated, positioned and oriented randomly, they would not have released counterions upon adsorbtion.

Let us return to the strongly correlated distribution of DNA. To complete the picture, we concentrate on the chemical potential of DNA rods. Corresponding equilibrium condition is similar to Eq. (位):

$$
\begin{array}{r}
L \eta^{*} \psi(0)=\left|\mu_{c}\right|+L\left(\eta^{*}-\eta_{c}\right)\left(k_{B} T / e\right) \ln \left(N_{1, s} / N_{1}\right) \\
-\left(L \eta^{* 2} / D\right) \ln \left(\lambda^{*} / a\right)+\left(L \eta_{c}^{2} / D\right) \ln \left(r_{s} / a\right),
\end{array}
$$

where $L$ is DNA length. In the right hand side, the first term, $\mu_{c} \simeq \mu_{W C}=-\left(L \eta^{* 2} / D\right) \ln \left(2 \pi \lambda^{*} / A\right)$, is the correlation chemical potential of DNA at the macroion surface. This is the energy of interaction of one DNA rod charged to $-\eta^{*}$ with its correlation hole which is a positive background stripe of width $A$. The second term is precisely the entropy gain due to the release of counterions. The third and fourth terms together represent the increase of the DNA self-energy when it moves from the bulk solution where it is charged to $-\eta_{c}$ and screened at $r_{s}$ to the surface where it is charged to $-\eta^{*}$ and screened at $\lambda^{*}$.

Fundamental equations (15), (16) are identical with Eqs. (57), (60) of Ref [14], which are derived there in a more rigorous way. The solution of these equations is given by Eqs. (10), (11), which simultaneously describe the correlation induced charge inversion and counterion release.

Let us now continue to explore what happens if we imagine that DNA is randomly distributed on the macroion surface with respect to both position and orientation of rods, i. e. if we artificially drop all correlation effects. As we already mentioned, Eq. (15) yields $\eta^{*}=\eta_{c}$ in this approximation. Furthermore, $\mu_{c}=0$ in the absence of correlations, so that Eq. (16) yields $\psi(0)=0$ and $\sigma^{*}=0$. Thus, assuming adsorbed DNA randomly distributed on the macroion surface with respect to both positions and orientations of rods, we arrive at the conclusion that there is neither counterion release nor charge inversion. By contrast, lateral correlations leading to parallel alignment of DNA molecules, can drive charge inversion even without counterion release, as this happens, e.g., for weakly charged $\mathrm{PE}$, for which $\eta_{0}<\eta_{c}$. In this sense, we conclude that correlations are the driving force of both counterion release and charge inversion.

Returning to the correlated distribution of DNA let us consider also a limit of very large $r_{s}$, which is not very realistic, but interesting from a theoretical standpoint. According to the Eq. (11), DNA net charge $\eta^{*}$ increases with increasing $r_{s}$, which physically means higher proportion of released counterions. To address this regime, we should make one step back including the $\ln \left(\eta_{0}-\eta^{*}\right) /\left(\eta_{0}-\eta_{c}\right)$ term in Eq. (15). For our purposes here it is sufficient just to approximate $\eta^{*}$ by $\eta_{0}$ in the Eq. (16). First and third term in the right-hand side combine then to $\left(L \eta_{0}^{2} / D\right) \ln (A / 2 \pi a)$, which vanishes at $A=2 \pi a$ and is negligible around this point. Therefore, the averaged surface potential in this case

$\eta_{0} \psi(0)=\left(\eta_{0}-\eta_{c}\right)\left(k_{B} T / e\right) \ln \left(N_{1, s} / N_{1}\right)+\left(\eta_{c}^{2} / D\right) \ln \left(r_{s} / a\right)$,

looks like the sum of counterion release and metallization terms. Interestingly, both of them are driven by correlations!

We conclude repeating that the underlying physics of charge inversion is always determined by correlations. An explicit treatment of correlations provides regular and universal description of this phenomenon. Apart from charge inversion, the correlations manifest themselves also in a number of other ways, including metallike properties of the macroion surface over certain range of length scales and including the release of counterions for the case of highly charged $Z$-ions, such as DNA.

T. T. N. and B. I. S. are supported by NSF DMR9985985. 
[1] J. Ennis, S. Marcelja and R. Kjellander, Electrochim. Acta, 41, 2115 (1996).

[2] T. Wallin, P. Linse, J. Phys. Chem. 100, 17873 (1996); 101, 5506 (1997).

[3] V. I. Perel and B. I. Shklovskii, Physica A 274, 446 (1999).

[4] B. I. Shklovskii, Phys. Rev. E 60, 5802 (1999).

[5] E. M. Mateescu, C. Jeppersen and P. Pincus, Europhys. Lett. 46, 454 (1999);

[6] S. Y. Park, R. F. Bruinsma, and W. M. Gelbart, Europhys. Lett. 46, 493 (1999);

[7] J. F. Joanny, Europ. J. Phys. B 9117 (1999).

[8] P. Sens, E. Gurovitch, Phys. Rev. Lett. 82, 339 (1999).

[9] R. R. Netz, J. F. Joanny, Macromolecules, 32, 9013 (1999).

[10] R. R. Netz, J. F. Joanny, Macromolecules, 32, 9026 (1999).

[11] Y. Wang, K. Kimura, Q. Huang, P. L. Dubin, W. Jaeger, Macromolecules, 32 (21), 7128 (1999).

[12] M. Lozada-Cassou, E. Gonzalez-Tovar, and W. Olivares, Phys. Rev. E 60, R17 (1999).

[13] T. T. Nguyen, A. Yu. Grosberg, B. I. Shklovskii, Phys. Rev. Lett. 85, 1568 (2000);

[14] T. T. Nguyen, A. Yu. Grosberg, B. I. Shklovskii, J. Chem. Phys. 113, 1110 (2000).

[15] R. Messina, C. Holm, K. Kremer, Phys. Rev. Lett. 85, 872 (2000); Euro. Phys. Lett., 51, 461 (2000).

[16] T. T. Nguyen, B. I. Shklovskii, Physica A, 293, 324 (2001).

[17] P. Chodanowski and S. Stoll, Macromolecules, (2001).

[18] T. T. Nguyen, B. I. Shklovskii, J. Chem. Phys. (2001).

[19] A. V. Dobrynin, A. Deshkovski and M. Rubinstein,
Macromolecules (2001).

[20] D. Andelman, J. F. Joanny, cond-mat/0011072

[21] K. B. Zeldovich, A. R. Khokhlov, Eur.Phys. J. E. (2001).

[22] M. Tanaka, A. Yu. Grosberg, J. Chem. Phys. (2001).

[23] L. G. Gulbrand, Bo Jonsson, H. Innerstrom, and P. Linse, J. Chem. Phys. 80, 2221 (1984); R. Kjellander and S. Marcelja, Chem. Phys. Lett. 114, 124(E) (1985); I. Rouzina and V. A. Bloomfield, J. Phys. Chem. 100, 9977, (1996); N. Gronbech-Jensen, R. J. Mashl, R. F. Bruinsma, and W. M. Gelbart, Phys. Rev. Lett. 78, 2477 (1997); Y. Levin, J. J. Arenzon, and J. F. Stilck, Phys. Rev. Lett. 83, 2680 (1999); B. I. Shklovskii, Phys. Rev. Lett. 82, 3268 (1999).

[24] N. D. Lang, Solid state physics, Vol. 28, Edited by H. Ehrenreich, F. Seitz, and D. Turnbull, Academic Press, New York (1973).

[25] L. D. Landau and E. M. Lifshitz, Quantum Mechanics (nonrelativistic theory), Chapter X, Oxford, Pergamon Press (1980).

[26] A. G. Moreira, R. R. Netz, Europhys. Lett.; condmat/0009376 (see also this volume).

[27] G. S. Manning, J. Chem. Phys. 51, 924 (1969).

[28] L. D. Landau and E. M. Lifshitz, Statistical Physics, Part 1, Chapter VII, Oxford, Pergamon Press (1977).

[29] T. T. Nguyen, B. I. Shklovskii, Phys. Rev. E (2001).

[30] T. P. Smith, W. I. Wang and P. J. Stiles, Phys. Rev. B34, 2995 (1986); M. S. Bello, E. I. Levin, B. I. Shklovskii, and A. L. Efros, Sov. Phys. JETP 53, 822(1981); A. L. Efros, Solid State Comm. 65, 1281 (1988); S. V. Kravchenko, D. A. Rinberg, S. G. Semenchinsky, and Pudalov, Phys. Rev. B42, 3741 (1990); J. P. Eisenstein, L. N. Pfeifer and K. W. West, Phys. Rev. Lett. 68, 674 (1992). 